\title{
Joint Spectrum Sensing and Transmission Strategy for Energy-Efficient Cognitive Radio Networks
}

\author{
$\mathrm{He} \mathrm{Li}^{*}$, Xinxin Feng*, Xiaoying Gan*, Zhongren $\mathrm{Cao}^{\dagger}$ \\ * Department of Electronic Engineering, Shanghai Jiao Tong University, Shanghai, 200240, China \\ ${ }^{\dagger}$ Information Sciences Institute, University of Southern California, Arlington, Virginia, 22203 \\ Email:\{plich, fxx1116, ganxiaoying\}@ sjtu.edu.cn, zcao@isi.edu
}

\begin{abstract}
In this paper, we consider a cognitive radio network in which a secondary user (SU) has opportunities to access one of multiple channels licensed by several primary users (PUs). Before transmission, SU should sequentially decide which channel to explore and in what sequence, as well as whether to access or to sleep. Our goal is to design a joint spectrum sensing and transmission strategy for $\mathrm{SU}$ to maximize energy efficiency, which is defined as the number of bits transmitted per unit energy consumption (including both transmission and operating energy). By modeling PUs' occupancy activities as a Markov process, we formulate the problem of designing optimal sensing and transmission strategy as a Partially Observable Markov Decision Process (POMDP). To solve it in a practical way, we establish the optimal threshold structure of myopic strategy and propose the algorithm to determine the optimal sensing order, which takes low computational complexity. Simulation results show that the energy efficiency loss of proposed approach is less than $8 \%$ compared with optimal solution. In addition, our approach can improve energy efficiency by roughly $15 \%$ to $\mathbf{3 5} \%$ compared to the traditional approaches, with less than $10 \%$ loss of capacity.
\end{abstract}

\section{INTRODUCTION}

With the rapid development of wireless communication, static spectrum allocation schemes cannot meet the requirements of wireless spectrum resource. For the purpose of improving spectrum efficiency, cognitive radio, which supports dynamic spectrum allocation, has been proposed [1]. In a cognitive radio network, secondary users (SUs) are allowed to opportunistically access the idle spectrums, which have been licensed to but not used by the primary users (PUs).

Regarding the growing attentions paid on global warming and climate change, the capability of energy saving [2] [3] turns into a significant metric to evaluate the performance of wireless communication networks. Meanwhile, mobile terminals are sensitive to energy saving capability which guarantees longer service time and richer service diversity [4]. With the development of large scale cognitive network, the activity of secondary users and the attributes of networks become much more complicated [5] [6]. Accordingly, energy-efficient network solutions, which can meet the demands of high capacity and low energy consumption simultaneously, have yielded a stream of new research activity.

Some works have been done on designing a cognitive radio network with the concern of energy efficiency. Cui et.al modeled the energy consumption of circuit and transmission in [7].
In the perspective of energy constraint, Chen et.al [8] designed a joint sensing and access strategy for opportunistic spectrum access based on POMDP model, which aimed at maximizing the throughput with energy constraint. In addition, Hoang et.al [9] extended Chen's works and discussed the optimal sensing duration, by which a tradeoff between achievable throughput and energy consumption was achieved. Pei et.al [10] proposed a more intuitive definition of energy efficiency and consider the optimal sensing strategy, power allocation, and sensing order problem. In that paper the occupancy activity of PUs is considered to be a pure random process and the idle probability is known to SU.

We consider a scenario, where SU has only limited information of channel states. To find spectrum opportunities as well as to provide protection to PUs, an SU must perform spectrum sensing before it transmits over a channel. An SU can conduct sensing and probing on each channel sequentially to gather information about network states and choose a channel for transmission. Based on this structure, an energy-efficient joint sensing and access strategy should be designed, which decides when to stop sensing and which channel to be used.

Different from the work in [10], we model PUs' occupancy activity as a Markov chain. Hereby we formulate the problem of joint sensing and transmission over multiple channels as a POMDP. We study myopic strategy to solve the problem in a practical way, and propose the algorithm to determine optimal sensing order with less computational complexity than dynamic programming based method. Another contribution is that, we introduce the capability of recall in sensing procedure. Accordingly, we prove that the optimal stopping rule of myopic strategy is a threshold based solution and obtain the closed form of threshold.

The rest of this paper is organized as follows. System model is described in Section II. The process of sensing and transmission is formulated as POMDP in Section III. Myopic strategy to solve the problem is discussed in Section IV. Simulation results and analysis are shown in Section V. Finally, conclusions are drawn in Section VI.

\section{SYSTEM MODEL}

We consider an opportunistic spectrum access (OSA) scenario where an SU must explore multiple channels before it exploits it. Both PUs' activities and channel fading conditions 


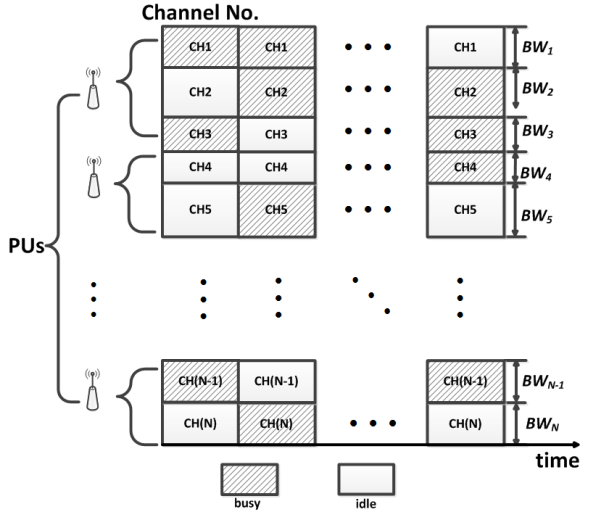

(a) Network model.

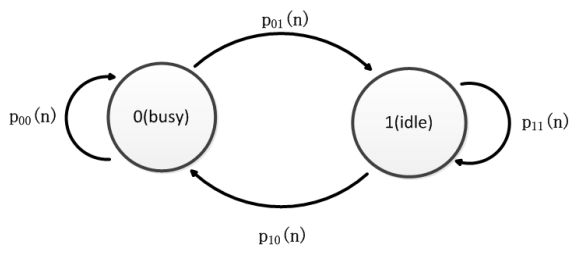

(b) Markov model of PU occupancy activity.

Fig. 1. Primary network.

are taken into consideration. An SU should exploit history information of these channels to maximize its energy efficiency by choosing proper actions.

\section{A. Primary Network}

As shown in Fig. 1, we study a cognitive network consisting of $N$ channels licensed by PUs, each with potentially different bandwidth $B W_{n}(n=1, \ldots, N)$. Each channel evolves as a two-state discrete-time Markov chain. The two states indicate the PUs' activities of idle (defined as state 1) and busy (defined as state 0 ). We denote the spectrum occupancy state as

$$
\mathbf{h}(t) \triangleq\left[h_{1}(t), \ldots h_{N}(t)\right] .
$$

The state transition probabilities of channel occupancy on the $n$th channel are given by $\left\{p_{i j}(n), i, j=0,1\right\}$, which are are independent to others and assumed to be known to SU.

\section{B. Secondary Network}

We assume that there is only one pair of SU transmitter and receiver who operates in an overlay manner with PUs. The SU can sense one channel at a time and access only one during a single transmission. We assume the channel states (including the occupancy states and the fading conditions) are unchanged within a frame, which consists of stages for sensing, probing and transmission.

Fig. 2 shows a single frame of SU, which consists of two phases: sensing \& probing phase, and data transmission phase.

In sensing and probing phase, the SU senses channels sequentially. If a channel is sensed idle, the SU will probes the

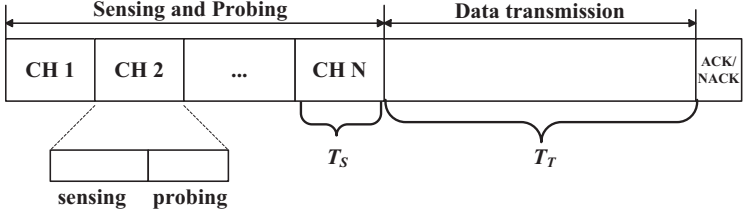

Fig. 2. Frame structure of $\mathrm{SU}$, with sensing order given as $\{1,2, \ldots, \mathrm{N}\}$.

channel to estimate the channel condition. SU can record all sensing and probing results. After several steps of sensing and probing operation, SU may decide to stop sensing and probing phase. When to stop this phase is decided by strategy, which will be discussed in the next several sections.

In data transmission phase, SU would choose to sleep to wait for better network status, or to start transmission directly. SU would get ACK for a successful transmission and NACK for an unsuccessful transmission. The sensing and probing time $T_{S}$ for a single channel and transmission time $T_{T}$ are assumed to be fixed. Whether to transmit and which channel is chosen to be transmit on is also decided by strategy, which will be discussed in the next several sections.

Spectrum sensing on each channel is a binary hypotheses test to determine between $\mathcal{H}_{0}\left(\right.$ i.e. $\left.h_{n}=0\right)$ and $\mathcal{H}_{1}\left(\right.$ i.e. $h_{n}=$ $1)$. With given sensing time and sensing algorithm, the energy cost $e_{S}$ of sensing and probing on each channel is also a fixed value in this paper. In addition, on the $n$th channel, we denote the probability of false alarm as $P_{f, n}$ and probability of detection as $P_{d, n}$.

\section{Energy Efficiency}

In this paper, energy efficiency is defined as

$$
\eta=\frac{\text { Average Number of Bits Transmitted }}{\text { Average Energy Consumed }}
$$

Energy consumption in the $m$ th frame may include the following two parts: 1) the energy consumed in the sensing and probing phase; 2) the energy consumed in the data transmission phase $E_{t x \_n}(m)$. Hereby, the overall energy cost is:

$$
E(m)=E_{t x_{-} n}(m) i_{T}(m)+n_{S}(m) e_{S}
$$

where $0 \leq n_{S}(m) \leq N$ is the number of channels sensed in the sensing and probing phase. $i_{T}(m) \in\{0,1\}$ is the indicator which indicates whether SU transmit in frame $m$ or not.

We assume the SU transmits at a fixed rate $R$, and adjusts its transmission power to ensure successful transmission. We denote $c$ as the speed of light, $d$ as the distance between the SU transmitter and the SU receiver, $f_{c, n}$ as the carrier frequency of channel $n, N_{0} / 2$ as the power spectral density of the noise, $N_{f}$ as the receiver noise figure, $L$ as the link margin compensating the hardware process variation and imperfection. On the $n$th channel the transmit power $P_{t_{-} n}$ can be written as:

$$
P_{t \_n}=\frac{S N R_{n} L \sigma_{n}^{2}}{\rho_{n} y_{n}}
$$


where $\rho_{n}=\left(\frac{c}{4 \pi d f_{c, n}}\right)^{2}$ captures the propagation loss, and $\sigma_{n}^{2}=N_{0} B W_{n} N_{f}$ is the noise power at the receiver front end. $y_{n}$ is the channel gain of the $n$th channel, which can be gotten by channel probing and its distribution is known to SU. $S N R_{n}$ is determined by transmission rate:

$$
S N R_{n}=\frac{2^{R / B W_{n}-1}}{\Gamma}
$$

where $\Gamma$ is considered as the SNR gap to channel capacity, which is determined by modulation type and BER requirement [7].

We can represent the energy consumption of transmission as [7]:

$$
E_{t x_{-} n}=P_{t x_{-} n} T_{T}=\left(\frac{\xi}{\zeta} P_{t_{-} n}+P_{c}\right) T_{T}
$$

where $\frac{\xi}{\zeta}$ is a fixed value determined by the property of power amplifier, and $P_{c}$ is the power consumed in circuits, which can also be considered as a fixed value.

\section{PROBLEM FORMULATION}

Within a frame, the SU may not sense all channels and there are sensing errors as well. Thus we can formulate the problem of designing optimal sensing and transmission strategy as a POMDP problem.

Network states and stages: The channel state of each channel at frame $m$ may comprise two parts of information, which are the spectrum occupancy state and the channel fading condition. We use $s_{n}(m)=\left(h_{n}(m), y_{n}(m)\right)$ to indicate the state of channel $n$ at frame $m$, and the network state at frame

\begin{tabular}{|c|c|c|c|c|c|c|}
\hline \multicolumn{7}{|c|}{ Frame $m$} \\
\hline \multicolumn{5}{|c|}{ Sensing and Probing } & \multicolumn{2}{|l|}{ Data transmission } \\
\hline $\mathrm{CH} a(1)$ & $\cdots$ & $\mathrm{CH} a(t)$ & $\cdots$ & $\begin{array}{c}\text { CH } \\
a\left(n_{S}(m)\right)\end{array}$ & $\mathrm{CH}\left|a\left(n_{S}(m)+1\right)\right|$ & $\mid \begin{array}{l}A \mathrm{ACK} / \\
\mathrm{NACCK}\end{array}$ \\
\hline \multirow[t]{2}{*}{ stage 1} & $\cdots$. & & $\therefore \cdots$ & stage $n_{S}(m)$ & stage $n_{S}(m)+1$ & \\
\hline & \multicolumn{3}{|c|}{ action a $(t)$ observation } & & & \\
\hline
\end{tabular}
$m$ can be denoted as $\mathbf{s}(m)=\left[s_{1}(m), \ldots, s_{N}(m)\right]$.

Fig. 3. Frame structure of SU, with sensing order given as $\{a(1), a(2), \ldots, a(N)\}$.

We divide the $m$ th frame into at most $(N+1)$ stages. Each stage denotes a single operation of sensing \& probing, transmission, or sleep. As shown in Fig. 3, frame $m$ contains $n_{S}(m)+1$ stages.

Actions and observations: As shown in Fig. 3, a single stage may contain two procedures: action and observation.

At the beginning of stage $t$, SU performs three kinds of actions: choose one channel to continue sensing, choose one channel for transmission or sleep to wait for better network states. We define the sensing and transmission actions at the $t$ th stage as $a(t)$, where $a(t)$ is an integer and $-N \leq a(t) \leq N$. If $a(t)<0$, SU will choose to transmit on channel $|a(t)|$. If $a(t)=0$, SU will choose to sleep. If $a(t)>0, \mathrm{SU}$ will choose to sense channel $a(t)$. At the $\left(n_{S}(m)+1\right)$ th stage, SU decides to transmit or sleep, which also indicates the end of sensing and probing phase in the $m$ th frame.

There are two kinds of observations for SU, which are sensing observation and transmission observation respectively. Sensing observation denotes the observation of whole network states through sensing and probing, while transmission observation is the acknowledgement of ACK or NACK received after a transmission.

Update of belief vector: At the beginning of each stage, the SU's statistical knowledge of the network state is provided by its action and observation history. Since the state variation is independent between channels, we denote a belief vector $\mathbf{b}(t)=\left[b_{1}(t), \ldots, b_{N}(t)\right]$, where $b_{n}(t)$ is the conditional probability (given the action and observation history) that the $n$th channel is idle. As all kinds of actions can incur observations, the belief vector change after the each stage.

At the beginning of stage $t+1$, the belief vector $\mathbf{b}(t+1)$ can be obtained by incorporating the action and their results in the $t$ th stage. We list all kinds of cases, where the belief vector is updated.

Case I: When the action conducted in the $t$ th stage is sleep, the belief vector $\mathbf{b}(t+1)$ will be updated at the beginning of stage $t+1$ based on Markovian model of PU traffic. That is,

$\forall n \in\{1, \ldots, N\}, b_{n}(t+1)=b_{n}(t) p_{11}(n)+\left(1-b_{n}(t)\right) p_{01}(n)$

Case II: When the action conducted in the $t$ th stage is transmission, the belief vector of the chosen channel $|a(t)|$ will be assured to be 1 or 0 according to whether the transmitter receives an ACK response. Then the belief vector is updated again according to (7) at the beginning of stage $t+1$.

Case III: When the action conducted in the $t$ th stage is continuing sensing, the belief vector of channel $a(t)$, which is the channel chosen to be sensed in the $t$ th stage, will be updated according to the sensing result, if the channel is sensed idle, we have

$$
b_{a(t)}(t)=1-P_{f, a(t)} .
$$

If the channel is sensed busy, we have

$$
b_{a(t)}(t)=1-P_{d, a(t)} .
$$

At the beginning of stage $t+1$, the belief vector will stay unchanged.

Object: Our object is to maximize energy efficiency of the secondary user over $M$ frames. We will firstly formulate transmission gain and energy cost in one frame. Then the objective function of this POMDP problem will be proposed.

In frame $m$, SU will finally choose channel $\left|a\left(n_{S}(m)+1\right)\right|$ for transmission. The transmission gain can be denoted as:

$$
B(m)=b_{\left|a\left(n_{S}(m)+1\right)\right|}\left(n_{S}(m)+1\right) R T_{T} i_{T}(m) .
$$

Substituting (4), (5), (6) into (3), we can obtain the energy cost in frame $m$ as: 


$$
\begin{aligned}
& E(m)=n_{S}(m) e_{S}+\left(\frac{\xi}{\zeta} P_{t_{a} a(m)}+P_{c}\right) T_{T} i_{T}(m) \\
& =n_{S}(m) e_{S}+\left(\frac{\xi\left(2^{R / B W_{a(m)}-1}\right) L \sigma_{a(m)}^{2}}{\zeta \Gamma \rho_{a(m)} y_{a(m)}}+P_{c}\right) T_{T} i_{T}(m)
\end{aligned}
$$

The object of this POMDP problem is to find an optimal policy $\pi$ which can maximize the total energy efficiency in $M$ frames. A policy $\pi$ is a sequence of functions $\pi=$ $\left[\mu_{1}, \mu_{2}, \ldots, \mu_{t}, \ldots\right]$, where $\mu_{t}$ maps each belief state $\mathbf{b}(t)$ to a sensing and transmission action $\{a(t)\}$. The optimal sensing and transmission strategy are given by

$$
\pi^{*}=\arg \max _{\pi} \mathbb{E}\left[\frac{\sum_{m=1}^{M} B(m)}{\sum_{m=1}^{M} E(m)} \mid \mathbf{b}(1)\right]
$$

where the initial belief vector $\mathbf{b}(1)$ can be set as the stationary distribution of channel occupancy state.

In order to obtain the optimal sensing and transmission strategy, we need to enumerate all possible states that SU may experience over $M$ frames. After that, we can obtain $\left\{n_{S}(1), \ldots, n_{S}(M)\right\},\left\{i_{T}(1), \ldots, i_{T}(M)\right\}$ for each strategy and calculate the corresponding objective function. Then we can search the path by which SU can obtain the maximum expected energy efficiency over $M$ frames.

However, there are at most $M(N+1)$ stages in $M$ frames, the searching space is relatively large and the computational complexity required to obtain the optimal strategy is very high, which makes it unfeasible to be implemented on SU's transmitter.

\section{MYOPIC SENSING AND TRANSMISSION STRATEGY}

One of the methods for solving the problem in a much more feasible way is to apply the myopic strategy [11], which only focuses on the immediate reward of the current frame. In the following section, we will study the myopic strategy in the $m_{0}$ th frame with a given belief vector $\mathbf{b}(1)$. Our object is to maximize energy efficiency in one frame. Thus sleep will never be an optimal choice for myopic strategy, $i_{T}\left(m_{0}\right)=1$.

At the beginning of stage $t_{0}$, with a given sensing order $[a(1), \ldots, a(N)]$, we denote $\eta_{t_{0},[a(1), \ldots, a(N)]}(t)$ as the maximum expected energy efficiency achieved on the condition that SU stop sensing at stage $t\left(t>t_{0}\right)$. As we only consider the energy efficiency in one frame, we can write the expression of $\eta_{t_{0},[a(1), \ldots, a(N)]}(t)$ as:

$$
\eta_{t_{0},[a(1), \ldots, a(N)]}(t)=\max _{n \in\{a(1), \ldots, a(t-1)\}} \mathbb{E}\left[\frac{b_{n}\left(t_{0}\right) R T_{T}}{E_{t x_{-} n}+t e_{S}}\right] .
$$

Note that $\forall t<t_{0}, E_{t x \_n}$ is determined through probing. Thus $\mathbb{E}\left[\frac{b_{n}\left(t_{0}\right) R T_{T}}{E_{t x_{n}}+t e_{S}}\right]=\frac{b_{n}\left(t_{0}\right) R T_{T}}{E_{t x} n+t e_{S}}$.

Hereby we formulate the problem of optimal myopic strategy. At the beginning of stage $t_{0}$, SU need to determine the sensing order $a^{*}=\left[a^{*}(1), \ldots a^{*}(N)\right]$ and stopping point $t^{*}$ which can maximize the expected energy efficiency:

$$
\begin{aligned}
& \left\{a^{*}, t^{*}\right\}_{t_{0}}=\arg \max _{t \geq t_{0}} \max _{n \in\{a(1), \ldots, a(t-1)\}} \mathbb{E}\left[\frac{b_{n}\left(t_{0}\right) R T_{T}}{E_{t x_{-}}+t e_{S}}\right] \\
& =\arg \max _{t \geq t_{0}} \eta_{t_{0},[a(1), \ldots, a(N)]}(t) .
\end{aligned}
$$

At the beginning of each stage, SU need to list all possible sensing order and stopping point pair, and calculate the expected energy efficiency according to (14). Then SU can search the optimal sensing order and stopping point. This process is completed with high computational complexity.

In the following part of this section, we will decompose the problem: analyze how to determine optimal sensing order and design the respective optimal stopping rule.

The first proposition will show the feature of optimal sensing order.

Proposition 1. At stage $t_{0}$, the channel which SU should sense to optimize energy efficiency is

$$
n^{*}=\arg \max \mathbb{E}\left[\frac{b_{n} R T_{T}}{E_{t x_{-} n}+t_{0} e_{S}}\right]
$$

Proof: We assume the optimal sensing order $a_{t_{0}^{+}}^{*}=$ $\left[a^{*}\left(t_{0}\right), \ldots a^{*}(N)\right]$.

If $t^{*}=\arg \max _{t_{0} \leq t^{\prime} \leq N} \eta_{t_{0}, a_{t_{0}^{+}}^{*}}\left(t^{\prime}\right)=t_{0}$. SU would stop at stage $t_{0}$. We should sense channel $n^{*}$ to ensure optimality.

If $t^{*}=\arg \max _{t \leq t^{\prime} \leq N} \eta_{t_{0}, a_{t_{0}^{+}}^{*}}\left(t^{\prime}\right) \neq t_{0}$ and $a^{*}\left(t^{*}\right)=n^{*}$, SU would stop at stage $t^{*}$. In this case we can change the sensing order of $a^{*}\left(t_{0}\right)$ and $a^{*}\left(t^{*}\right)$ to obtain a sensing order with higher or equal energy efficiency. Thus we could sense $n^{*}$ at stage $t_{0}$ without loss of optimality,

If $t^{*}=\arg \max _{t \leq t^{\prime} \leq N} \eta_{t_{0}, a_{t_{0}^{+}}^{*}}\left(t^{\prime}\right) \neq t_{0}$ and $a^{*}\left(t^{*}\right) \neq n^{*}$, SU would stop at stage $t^{*}$. We can sense channel $n^{*}$ at stage $t_{0}$ without loss of optimality.

Proposition 1 shows that at the beginning of each stage, SU can make sure which stage to sense. In addition, as shown in (15), the next channel to be explored is not affected by sensing history. Thus at the beginning of the first stage, SU can determine the optimal sensing order of the whole frame.

We now present the detailed algorithm to determine sensing order according to Proposition 1. As shown in Algorithm 1, the computation complexity is only $\mathcal{O}\left(N^{2}\right)$, which is much less than $\mathcal{O}(N ! N)$, the dynamic programming based solution proposed in [10].

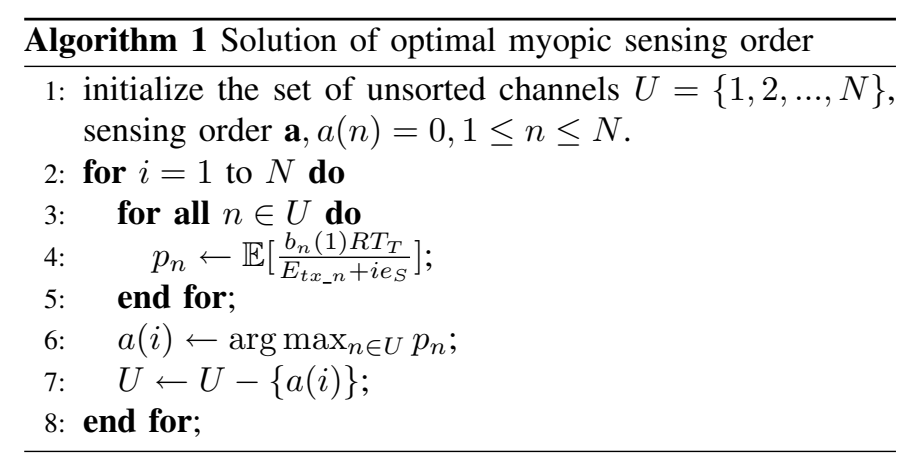


We will show that the optimal stopping rule is a threshold based structure through the following proposition.

Proposition 2. With the optimal order $[a(1), a(2), \ldots a(N)]$, at stage $t_{0}$, if

$$
\begin{aligned}
& \max _{n \in\left\{a(1), \ldots a\left(t_{0}-1\right)\right\}} \frac{b_{n}\left(t_{0}\right) R T_{T}}{E_{t x} n+\left(t_{0}-1\right) e_{S}} \geq \\
& \max _{n \in\{a(1), \ldots a(N)\}} \mathbb{E}\left[\frac{b_{n}\left(\bar{t}_{0}\right) R T_{T}}{E_{t x_{-} n}+t_{0} e_{S}}\right],
\end{aligned}
$$

then SU should choose to stop sensing to achieve maximum energy efficiency. Otherwise SU should continue sensing. we have

Proof: Suppose (16) is satisfied. Then for any $t^{\prime}>t_{0}$,

$$
\begin{aligned}
& \eta_{t_{0},[a(1), \ldots, a(N)]}\left(t^{\prime}\right)=\max _{n \in\left\{a(1), \ldots a\left(t^{\prime}-1\right)\right\}} \mathbb{E}\left[\frac{b_{n}\left(t_{0}\right) R T_{T}}{E_{t x_{-} n}+\left(t^{\prime}-1\right) e_{S}}\right] \\
& \leq \max _{n \in\left\{a(1), \ldots a\left(t^{\prime}-1\right)\right\}} \mathbb{E}\left[\frac{b_{n}\left(t_{0}\right) R T_{T}}{E_{t x}+t_{0} e_{S}}\right] \\
& \leq \max _{n \in\{a(1), \ldots a(N)\}} \mathbb{E}\left[\frac{b_{n}\left(t_{0}\right) R T_{T}}{\left.E_{t x_{n} n+t_{0} e_{S}}\right]}\right] \\
& \leq \max _{n \in\left\{a(1), \ldots a\left(t_{0}-1\right)\right\}} \frac{5_{n}\left(t_{0}\right) R T_{T}}{E_{t x_{-} n}+\left(t_{0}-1\right) e_{S}} \text {. }
\end{aligned}
$$

As shown in (17), for any $t^{\prime}>t_{0}$, stopping at stage $t^{\prime}$ will not achieve higher energy efficiency.

If (16) does not hold good, we have:

$$
\begin{aligned}
& \eta_{t_{0},[a(1), \ldots, a(N)]}\left(t_{0}\right)= \\
& \max _{2}\left\{\max _{n \in\left\{a(1), \ldots a\left(t_{0}-1\right)\right\}} \frac{b_{n}\left(t_{0}\right) R T_{T}}{E_{t x}+t_{0} e_{S}}, \max \mathbb{E}\left[\frac{b_{n}\left(t_{0}\right) R T_{T}}{E_{t x_{-} n}+t_{0} e_{S}}\right]\right\} \\
& =\max _{n \in\{a(1), \ldots a(N)\}} \mathbb{E}\left[\frac{b_{n}\left(t_{0}\right) R T_{T}}{E_{t x}+t_{0} e_{S}}\right] \\
& >\max _{n \in\left\{a(1), \ldots a\left(t_{0}-1\right)\right\}} \frac{b_{n}\left(t_{0}\right) R T_{T}}{E_{t x_{-} n}+\left(t_{0}-1\right) e_{S}}
\end{aligned}
$$

As shown in (18), sensing $a\left(t_{0}\right)$ at stage $t_{0}$ will achieve higher energy efficiency. Thus the proposition is proved.

Proposition 2 shows that at the beginning of each stage, SU can check (16) to decide whether to continue sensing. If SU stops at stage $t^{\prime}$, then we have $n_{S}\left(m_{0}\right)=t^{\prime}-1$.

\section{Simulation Results}

In this section we present the simulation results to evaluate the performance of our proposed algorithm. Simulation of parameters are listed in Table I.

\section{A. Energy Efficiency Evaluation}

Firstly, we evaluate the performance of proposed algorithm by comparing it with the other three approaches: Omniscience, sensing in fixed order and full sensing. In omniscience approach, SU has full information about the network status and the instantaneous channel gain of each channel. Thus SU can make the optimal choice directly. In sensing in fixed order approach SU has no information about network status. SU senses the channel in a fixed order and transmit on the first channel detected idle. In full sensing approach, SU will explore the whole network status and then decide which channel to access.

Fig. 4 illustrates the energy efficiency versus number of frames. In this case, the number of channels is $N=10$. Our proposed algorithm finally achieves an energy efficiency of 46Mbits/J.
TABLE I

Simulation PARAMETERS

\begin{tabular}{|l|l|}
\hline $\begin{array}{l}\text { Energy of sensing and probing } \\
e_{S}\end{array}$ & $5 \mathrm{~mJ}$ \\
\hline Bandwidth $B$ & $5 \mathrm{MHz}$ \\
\hline Pre-set data rate $R$ & $10 \mathrm{Mbps}$ \\
\hline Link margin $L$ & $10 \mathrm{~dB}$ \\
\hline Distance $d$ & $200 \mathrm{~m}$ \\
\hline Circuit power $P_{c}$ & $200 \mathrm{~mW}$ \\
\hline Sensing and probing time $T_{S}$ & $20 \mathrm{~ms}$ \\
\hline Transmission time $T_{T}$ & $100 \mathrm{~ms}$ \\
\hline Parameters of sensing errors & $P_{d}=0.95, P_{f}=0.05$ \\
\hline SNR gap $\Gamma$ & $\begin{array}{l}F o r M-Q M M, B E R=10^{-5} \\
\Gamma \approx-\ln (5 B E R) / 1.5=6.6023\end{array}$ \\
\hline Carrier frequency $f_{c, n}$ & $f_{c, n}=595+5 n(M H Z)$ \\
\hline PAR over drain efficiency $\xi / \zeta$ & 11.3745 \\
\hline Transition probabilities of PU & $\begin{array}{l}p_{01}(n)=0.3+0.6 * n / N \\
p_{10}(n)=0.8-0.6 * n / N\end{array}$ \\
\hline SU channel condition $y_{n}$ & $\begin{array}{c}\{1,2,3,4,5\} \text { with probability } \\
\{0.4,0.3,0.15,0.09,0.06\}\end{array}$ \\
\hline
\end{tabular}

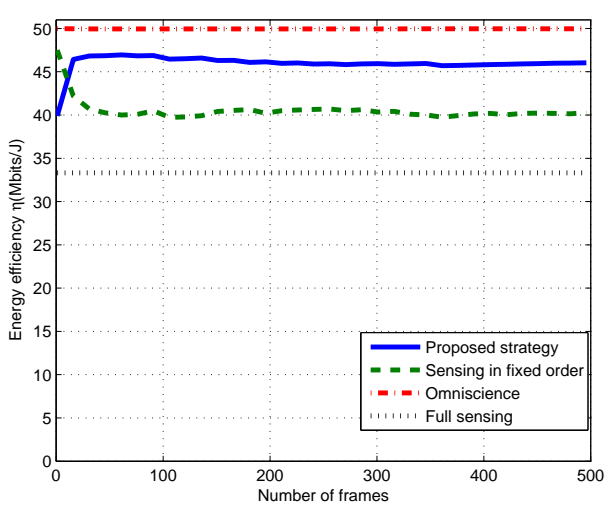

Fig. 4. Energy efficiency vs number of frames.

Note that the omniscience approach performs better than optimal POMDP solution because it involves no cost for sensing and probing. While our proposed algorithm performs only $8 \%$ worse than the omniscience approach, which implies that its loss of energy efficiency is less than $8 \%$ compared to the optimal POMDP solution.

With the capability to explore better channels, our proposed approach performs $15 \%$ better than fixed order approach. It also performs $35 \%$ better than full sensing approach by saving energy consumed in the sensing and probing phase through optimal sensing order.

\section{B. Effect of number of channels}

We show the energy efficiency achieved for different number of channels, compared with the other three approaches in Fig. 5. We can observe that for different number of channels, the achieved energy efficiency by our proposed algorithm is nearly constant around $46 \mathrm{Mbits} / \mathrm{J}$, which performs better than fixed order approach as a result of exploring better channels through the threshold structure and optimal sensing order. 


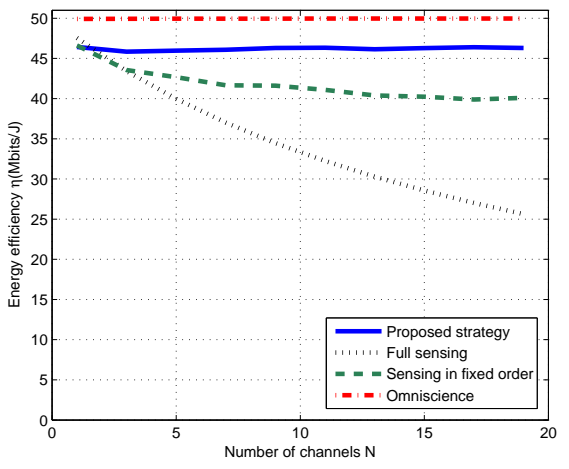

Fig. 5. Energy efficiency vs number of channels N.

Full sensing approach is sensitive to the number of channels, because the energy consumed in sensing and probing phase increases when number of channels $N$ increases. It performs $46 \%$ worse than our proposed method when $N=19$. As $N$ increases the gap of energy efficiency between full sensing approach and proposed approach also increases. This is because our proposed approach can explore the best channel faster through optimal sensing order.

\section{Energy efficiency - capacity tradeoff}

Fig. 6 illustrates the normalized average capacity and energy efficiency versus the sensing energy $e_{S}$ of the proposed strategy and the traditional throughput maximizing approach. In throughput maximizing approach, SU would sense channels in descending order of $\left\{b_{n}(t)\right\}$, and choose the first channel detected idle for transmission.

It shows that with the increase of $e_{S}$, the average energy efficiency of two approaches goes down. While as $e_{S}$ increases, the advantage of proposed strategy over traditional ones decreases. This is because that as sensing energy weighes more, the advantage of proposed strategy through picking channels with high energy efficiency weighes less. Our proposed strategy can perform at most $50 \%$ better on energy efficiency, and achieve over $90 \%$ capacity of the tradition one at the same time.

In practical situations power cost of sensing is usually less than $1 \mathrm{~W}$ and the time cost is usually less than $10 \mathrm{~ms}$ [7], which means that $e_{S}$ is usually less than $10 \mathrm{~mJ}$. As shown in Fig. 4, when $e_{S} \in[0,10 \mathrm{~mJ}]$, our approach can perform at least $30 \%$ better than traditional approach.

\section{CONCLUSION}

In this paper, we analyze the problem of joint sensing and transmission strategy in a multi-channel system. We formulate the energy efficiency maximization problem as a POMDP problem. In order to solve it in a more practical way, we discuss the myopic strategy. We reveal the feature of myopic strategy, propose the low complexity algorithm constructing optimal myopic sensing order, and derive the threshold based stopping rule as well as corresponding thresholds. Simulation results show the proposed algorithm does not bring principle

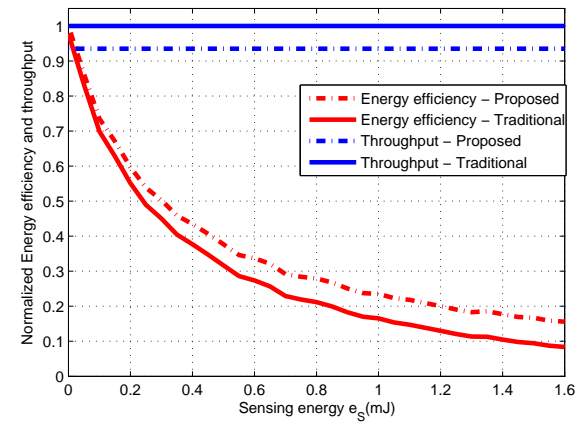

Fig. 6. Normalized energy efficiency \& capacity vs sensing energy $e_{S}$.

degradation compared with optimal solution, and achieves great improvement over traditional throughput maximization approach with acceptable capacity loss.

\section{ACKNOWLEDGMENT}

This work has been supported in part by the National Science Foundation of China under Grant 61102052, and Grant 60972050, by the National Fundamental Research Grant 2010CB731803, by the China Ministry of Education Fok Ying Tung Fund under Grant 122002, and by the NationalKey Project of China under Grant 2010ZX03002- 007-01, and Grant 2010ZX03003-001-01.

\section{REFERENCES}

[1] J. Mitola and G. Q. Maguire, "Cognitive radio: Making software radios more personal," Personal Communications,IEEE, vol. 6, pp. 13-18, 1999.

[2] A. Dejonghe, B. Bougard, S. Pollin, J. Craninckx, A. Bourdoux, L. V. der Perre, and F. Catthoor, "Green reconfigurable radio systems," Signal Processing Magazine,IEEE, pp. 90-101, May 2007.

[3] P. J, "Cognitive radio: An enabling technology for the green radio communications concept," IWCMC'09, pp. 21-24, June 2009.

[4] L. V. der Perre, W. V. Thillo, A. Dejonghe, and J. V. Driessche, "Tomorrow's wireless communication requires higher throughput and a smaller energy budget," Chip Design Magazine, 2010.

[5] W.Huang and X.Wang, "Capacity scaling of general cognitive networks," IEEE/ACM Transactions on Networking, vol. 20, no. 5, pp. 1501-1513, 2012.

[6] S. J. X.Wang, W.Huang and C.Hu, "Delay and capacity tradeoff analysis for motion cast," IEEE/ACM Transactions on Networking, vol. 19, no. 5, pp. 1354-1367, Oct 2011.

[7] S. Cui, A. J.Goldsmith, and A. Bahai, "Energy-constrained modulation optimization," IEEE transactions on wireless communications, pp. 23492360, Sep 2005.

[8] Y.Chen, Q.Zhao, and A.Swami, "Distributed spectrum sensing and access in cognitive radio networks with energy constraint," IEEE transactions on Signal Process, vol. 57, pp. 783-797, 2009.

[9] A. T. Hoang, Y.-C. Liang, W. D., Y. Zeng, and R. Zhang, "Opportunistic spectrum access for energy-constrained cognitive radios," Wireless Communications, IEEE Transactions on, vol. 8, pp. 1206-1211, Mar 2009.

[10] Y. Pei, Y.-C. Liang, K. C. Teh, and K. H. Li, "Energy-efficient design of sequential channel sensing in cognitive radio networks: Optimal sensing strategy, power allocation, and sensing order," Selected Areas in Communications, IEEE Journal on, vol. 29, pp. 1648-1659, Aug 2011.

[11] S.Ahmad, M.Liu, T.Javidi, Q.Zhao, and B.Krishnamachari, "Optimality of myopic sensing in multichannel opportunistic access," IEEE Trans. Inf. Theory, vol. 55, p. 4040C4050, 2009. 